\title{
Geographical distribution of observational activities for astronomy
}

\author{
A. Heck \\ Observatoire Astronomique, 11 rue de l'Université, F-67000 Strasbourg, France \\ e-mail: heck@astro.u-strasbg.fr
}

Received November 14; accepted December 2, 1997

\begin{abstract}
The geographical distribution of astronomyrelated observational activities is illustrated from comprehensive and up-to-date samples of professional institutions, of public observatories and planetariums, and of associations. Results are commented and, in particular, the lack of evolution over the past century in the overall distribution is pointed at as an alarming indicator.
\end{abstract}

Key words: sociology of astronomy - astronomical databases: miscellaneous

\section{Introduction}

The data used in the present study have been extracted from the master files for StarGuides (Heck 1997a) and StarWorlds (Heck et al. 1994), the latter one being the WWW version of the former one which is a classical directory on paper. The web version is itself the follow-on of on-line yellow-page services established previously at ESA/ESRIN (StarWays - see Heck et al. 1992) and at ESO (StarGates - see Albrecht \& Heck 1992). It is also one of the three components of the StarPages (see e.g. Heck 1997b) including in addition a database of personal web pages (StarHeads - see Heck 1995) and a huge dictionary of acronyms focused on astronomy, space and related fields and activities (StarBits - see Heck et al. 1994).

The expertise built up over now almost a quarter of a century in this kind of compilations and services (involving authentication and quality checking schemes together with a permanent updating process), as well as the overall stability of the master files, guarantee an excellent exhaustivity of the entries and an homogeneous coverage of the practical data gathered together. They are certainly the best sources available today for the study at hand. It is appropriate to recall here that, contrary to most on-line resources, StarWorlds is not only WWW-oriented.

Send offprint requests to: A. Heck

\section{The data}

At the time of writing, there were 6080 entries in the master files, out of which about a thousand were simple crosspointers. Thus the total number of effective organizations gathered together was 5086 with a total of 1504 sets of coordinates available.

It is interesting to notice that, in spite of the fact that sets of coordinates have been explicitly requested on our questionnaires sent for many years to all the organizations approached, less than a third of them registered at least one position. There are of course plenty of good explanations for that effect: institutions carrying out only theoretical research, associations badly located or without enough financial resources to afford observational means, and so on. Also the basic activities of a substantial number of organizations included in the resource are totally extraneous to observing. This is particularly the case for publishers, retailers, manufacturers, software producers, and so on. What is sure though is that providing coordinates in that context is a positive sign of a deliberate observational policy from the corresponding organizations.

It seemed appropriate to sort out the entries in three categories for this study: institutions, public observatories and planetariums, and finally associations. The first one is clearly related to professional astronomers. The two latter ones deal mainly with amateur astronomers and the grand public. They have strongly similar educational missions. The two last categories could of course be merged for a statistical investigation such as the current one, but it should be kept in mind that public observatories and planetariums have a more official structure and a better stability (mainly because of the buildings, equipment, and personnel involved) than most associations, the nature of which is consequently different.

Out of a total of 1857 institutions registered at the time of writing, we retained 610 of them who provided coordinates of observing sites. As quite a few of them had several observing sites, the total number of coordinates sets retained for that group was 816 . Not all institutions with coordinates were selected: from the registered profile 
of activities, we did not take for instance those exclusively involved in aeronomy, but we did include those studying the solar-terrestrial relationships, and so on. Some borderlines had to be adopted and we did it by making the best usage of the information provided by the organizations themselves (as part of the authentication/verification process, we request a report of activities).

There were 739 entries registered as public observatories and planetariums (not unfrequently both of them together) in the master files, out of which 269 (36\%) provided coordinates. The total number of locations for this category went up to 295 by including secondary sites. This is above average which can be understood since public observatories are, by definition, places where observations are logically conducted. In this category, 299 entries were purely public observatories and 183 of them (61\%) provided coordinates.

Out of the 1327 entries registered as associations in the master files, only 306 (23\%) of them provided coordinates of observing sites, well below the overall average. With the secondary sites, a total of 355 locations was reached. An obvious conclusion from these figures is that many associations have no observing sites or at least no fixed observing site, but of course members might have their own private facilities, not necessarily considered as being under the responsibility of the association. Some associations are also managing public observatories and planetariums, or they are using their sites, so there might be some overlap, but this is true for only a small number of them and it does not affect the statistical results (the corresponding locations are anyway superimposed on the maps).

\section{The maps}

We are presenting here the maps (drawn via IDL) that appeared to be the most interesting. Others can be produced of course and they can be requested from the author.

Figures 1 to 4 give the world distribution of institutions with blowups for Europe and North America, together with a stereographic view from the South Pole. Notice in particular the observing spots in Antarctica.

Figures 5 to 7 show the world distribution of public observatories and planetariums with enlargements for Europe and North America. Figures 8 to 10 provide the same for associations.

\section{Comments}

When dealing with this kind of statistical distributions, it is important to refrain from pushing the analysis of the data too far and to stay at an appropriate level: a global analysis, rather than a location-per-location perusal.

Apart from strong densities in Europe and the Eastern half of the U.S.A., the most striking feature - common to

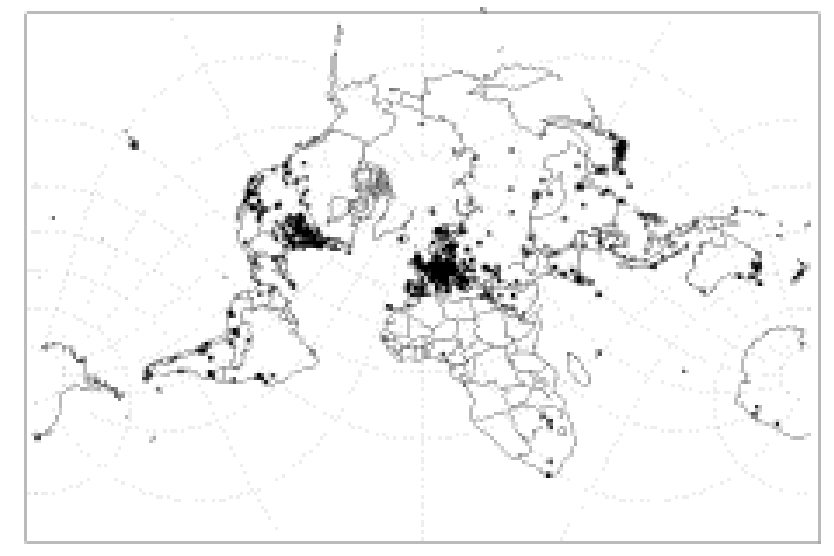

Fig. 1. World distribution of institutions (see text)

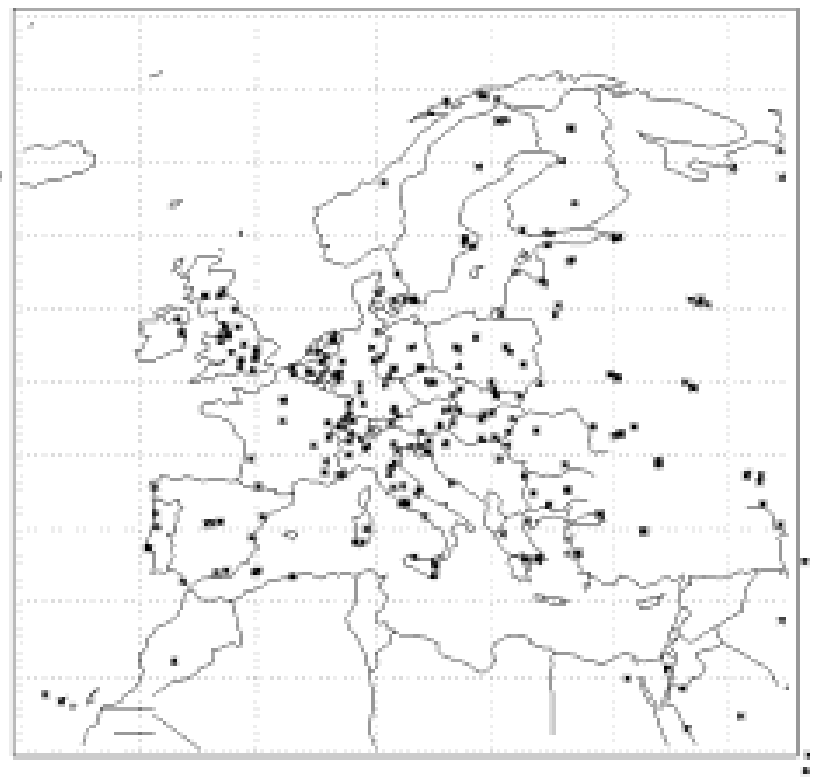

Fig. 2. European distribution of institutions (see text)

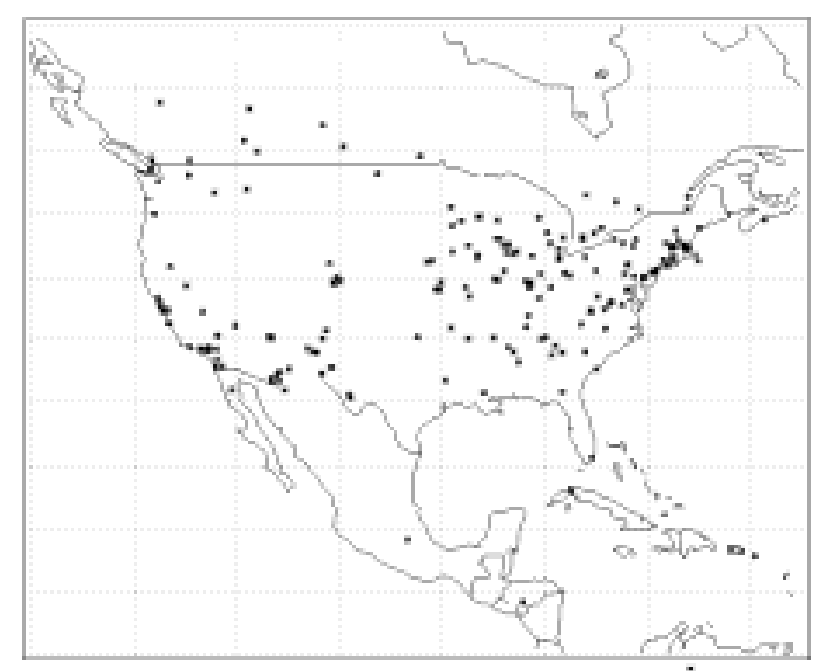

Fig. 3. North American distribution of institutions (see text) 


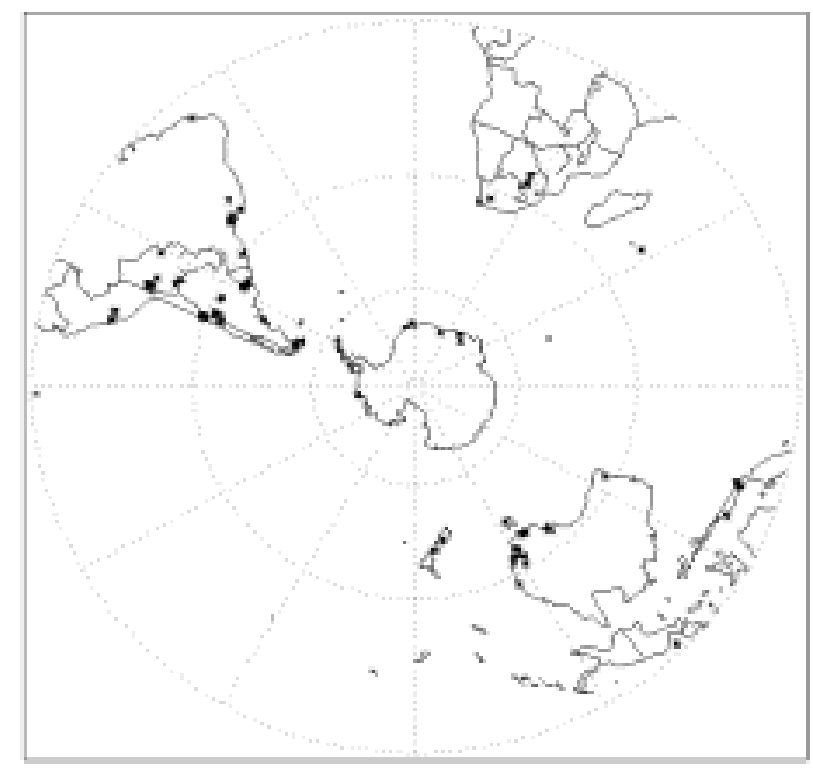

Fig. 4. Southernmost distribution of institutions (see text)

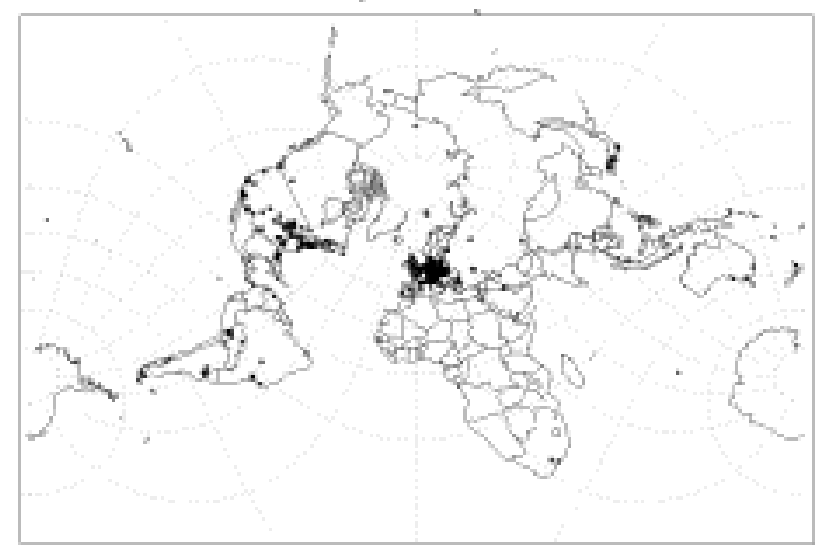

Fig. 5. World distribution of public observatories and planetariums (see text)

all categories - is the desperate emptiness of most of the African continent. This situation did not improve since we published earlier maps (Heck 1991) and such a persistence is really disturbing. A similar comment is of application to most of the so-called third-world countries. Astronomical activities are certainly linked to a comparatively wealthy level of evolution (in the socio-historical context) reached by countries or societies who have always more urgent priorities to be satisfied first. What is really alarming though, is that, if we compare the distributions presented here with that of Stroobant et al. (1907) reprinted in Heck (1991), the overall aspect did not change significantly over the past century. This should be a real concern not only for every astronomer, but well beyond our science, because it tells certainly something much more fundamental about long-term higher education in those parts of the world and

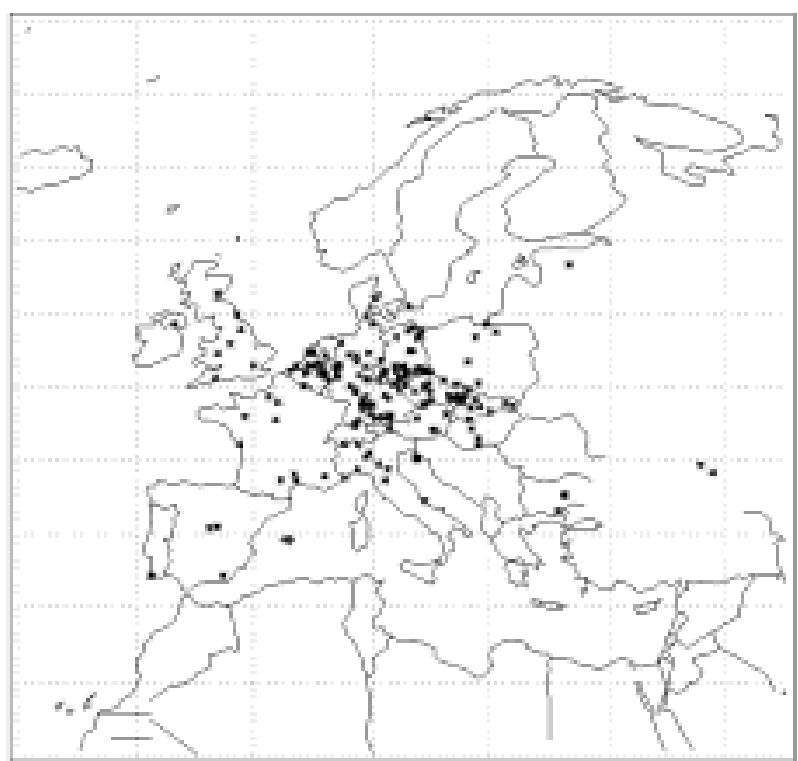

Fig. 6. European distribution of public observatories and planetariums (see text)

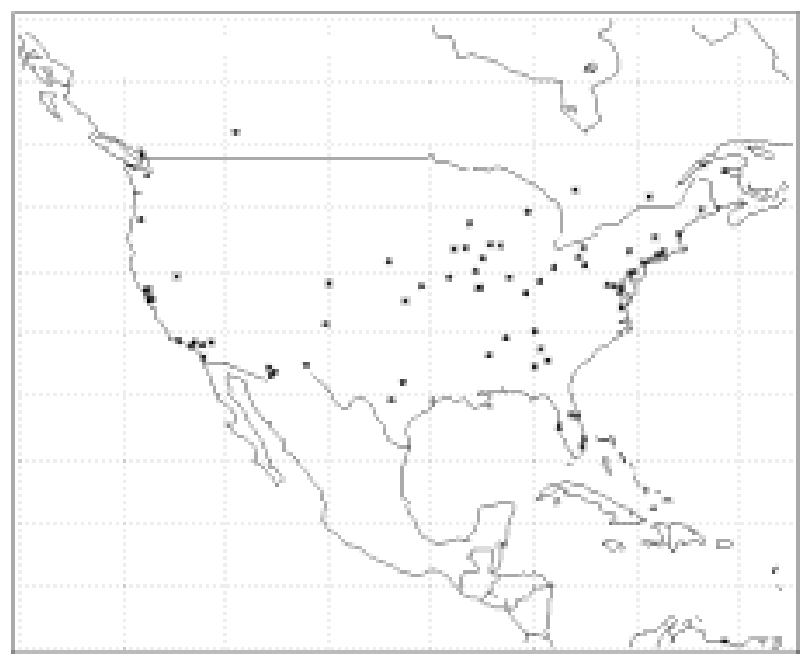

Fig. 7. North American distribution of public observatories and planetariums (see text)

on our way to deal with it even if, over the same range of time, a few additional observing facilities have been built in propitious parts of the world, especially in the Southern hemisphere.

The general aspects of the corresponding distributions between the three categories retained in this study are similar, with some nuances. In Europe for instance, France has comparatively less professional observing facilities and public observatories, but the difference disappears at the level of associations. The density of public observatories in the Netherlands, Germany, the Czech and Slovak Republics 


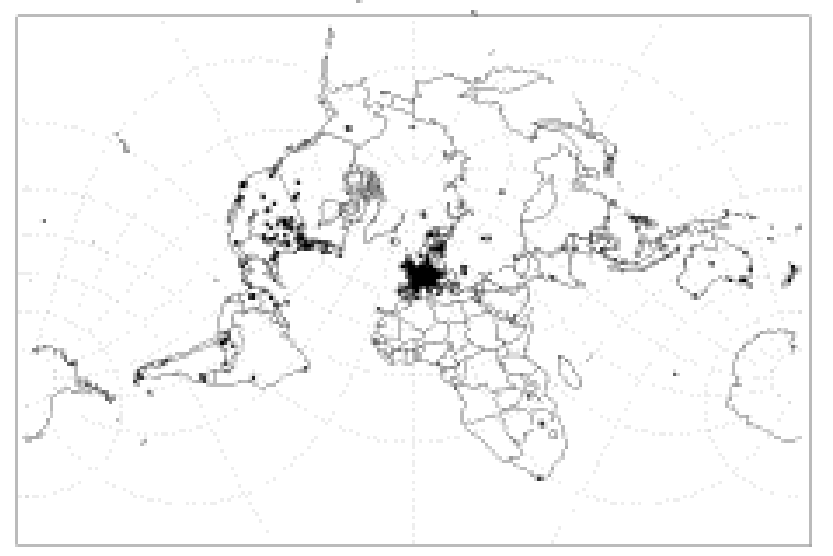

Fig. 8. World distribution of associations (see text)

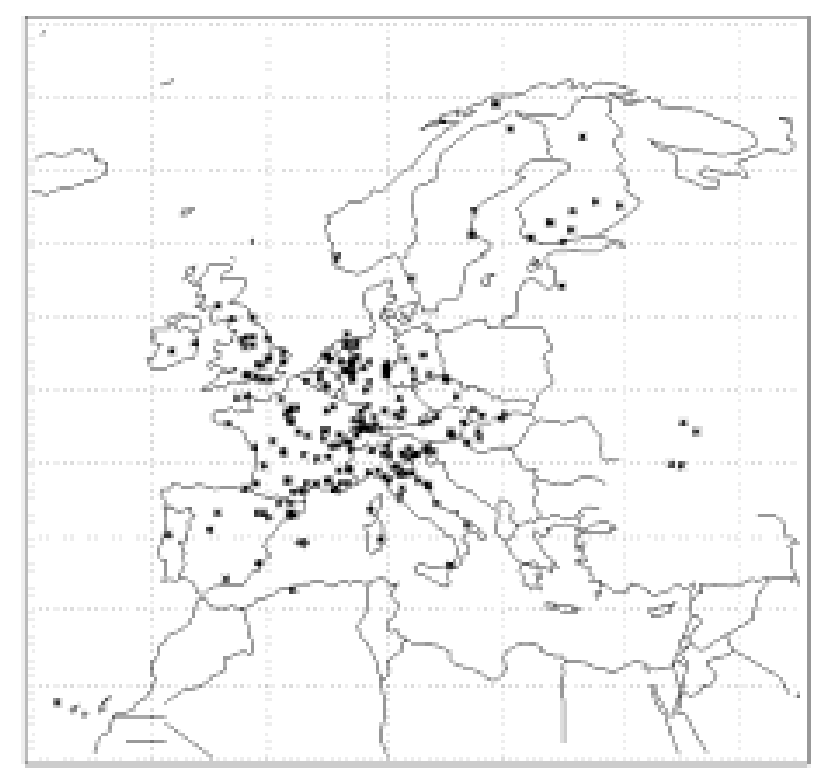

Fig. 9. European distribution of associations (see text)

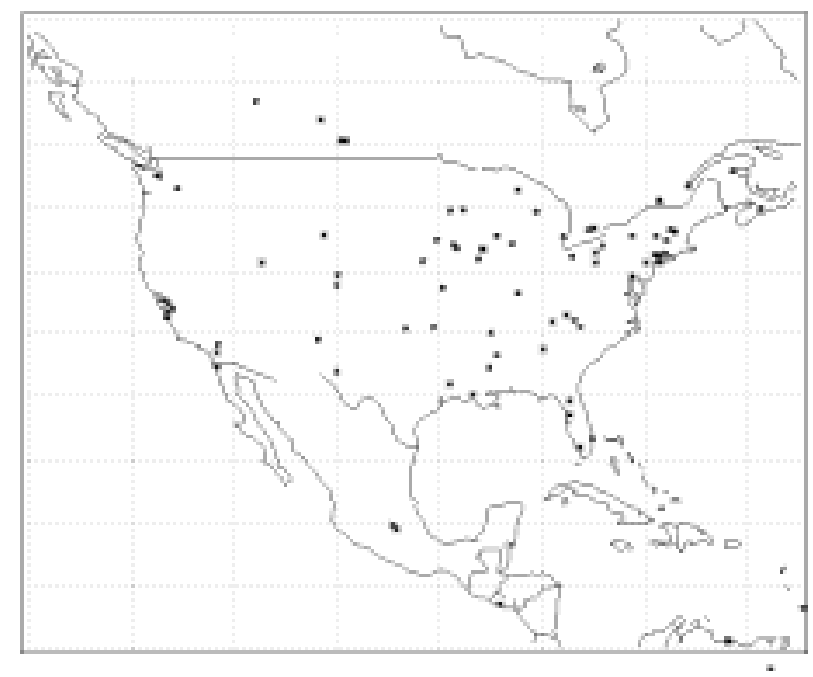

Fig. 10. North American distribution of associations (see text) testifies of cultural components and/or deliberate policies. Knowing the dynamism, facilities, and the generally wealthy level in the US, one would expect more observing sites linked to public observatories and associations, but - and this is another cultural component more is carried out there at the individual level as examplified in each issue of journals such as Astronomy or Sky E Telescope.

The distributions of public observatories, planetariums and associations has to be seen as much more than anecdotical. When we were publishing two separate directories for the professional institutions and for the associations (respectively IDPAI and IDAAS, predecessors of the StarGuides - see Heck 1991), many professional institutions were purchasing a copy of the second one. Apart from a standard library acquisition, identified purposes were the organization of observational campaigns involving amateurs round the world and strong collaborations for educational activities as well as interface with the public and with official bodies. Also, in these times of restricted funding for fundamental sciences, the critical importance of all these organizations must be - more than ever - fully appreciated.

Acknowledgements. We are very grateful to all persons and organizations who contributed over the past quarter of the century to the very substance of the master files used here by returning the questionnaires, by providing the relevant documentation, by participating in the various procedures of maintenance, validation and verification of the information, or otherwise. Our thanks go also to Emmanuel Chereul and to Ilunga Mwana Umbela for their kind introduction to IDL's mapping capabilities.

\section{References}

Albrecht M.A., Heck A., 1994, A\&AS 103, 471

Heck A., 1991, Astronomical directories, in Databases \& OnLine Data in astronomy, Albrecht M.A. \& Egret D. (eds.). Kluwer Acad. Publ., Dordrecht, p. 211-224

Heck A., 1995, A\&AS 109, 265 (see also the URL: http://vizier.u-strasbg.fr/starheads.html)

Heck A., 1997a, StarGuides 1997 - A directory of astronomy, space sciences and related organizations of the world, Publ. Spéc. CDS 29, viii + pp. 1022 (ISBN 2-908064-27-8)

Heck A., 1997b, Electronic yellow-page services: The Star*s Family as an example of diversified publishing, in Electronic Publishing for Physics and Astronomy, Heck A. (ed.). Kluwer Acad. Publ., Dordrecht, p. 221-220 (see also the URL: http://vizier.u-strasbg.fr/starpages.html)

Heck A., Ciarlo A., Stokke H., 1992, A\&AS 96, 565-566

Heck A., Egret D., Ochsenbein F., 1994, A\&AS 108, 447-448 (see also the URLs: http://vizier.u-strasbg.fr/ starworlds.html and http://vizier.u-strasbg.fr/starbits. html)

Stroobant P., Delvosal J., Philippot H., Delporte E., Merlin E., 1907, Les observatoires astronomiques et les astronomes, Hayez, Bruxelles, p. 318 